\title{
Fantastic plastic
}

\author{
Flexible and stretchable solar cells and light-emitting diodes are respectively perceived as being next- \\ generation photovoltaic and lighting technologies, and are attracting the attention of the general public.
}

Polymer (or 'plastic') electronic and optoelectronic components have many advantages over their inorganic counterparts, including being lightweight, flexible, easy to integrate, and inexpensive to manufacture, and having a low environmental impact during fabrication and operation. They offer unique attractions and opportunities, and can be used to realize devices for applications in energy generation, displays, lighting and biomedicine. Solar cells and light-emitting diodes (LEDs) produced from organic polymers and molecules can be fabricated using inexpensive roll-to-roll technologies (similar to those used for printing newspapers) that are amenable to large-scale production. Such devices have been extensively researched and are now beginning to be commercialized.

The growing interest in polymer electronics and photonics was evident at the Seventh International Conference on Materials for Advanced Technologies (ICMAT 2013) held in Singapore from 30 June to 5 July. In particular, a large technical session was devoted to polymer organic semiconductor materials for photovoltaics and LEDs, and a plenary talk was dedicated to polymer solar cells.

The plenary talk was given by Alan Heeger, a 2000 Nobel Laureate in Chemistry from the University of California, Santa Barbara, USA. He explained how the Heisenberg uncertainty principle affects bulk heterojunction solar cells. He also described the discovery of ultrafast photoinduced electron transfer as being the scientific foundation for a technology for producing low-cost plastic solar cells. By performing a series of transient absorption measurements, his group demonstrated that the uncertainty principle can enable ultrafast ( $<100 \mathrm{fs}$ ) charge transfer over distances of 10-20 nm in the nanoscopically textured material in bulk heterojunction solar cells. Because the spatial extent of the initially photoexcited wavefunction is determined by the uncertainty principle, and not by the eigenfunctions of the Schrödinger equation describing the disordered nanostructured material, his group anticipates the emergence of new physics on the nanoscale.

Also at the conference, Richard Friend from the University of Cambridge in the

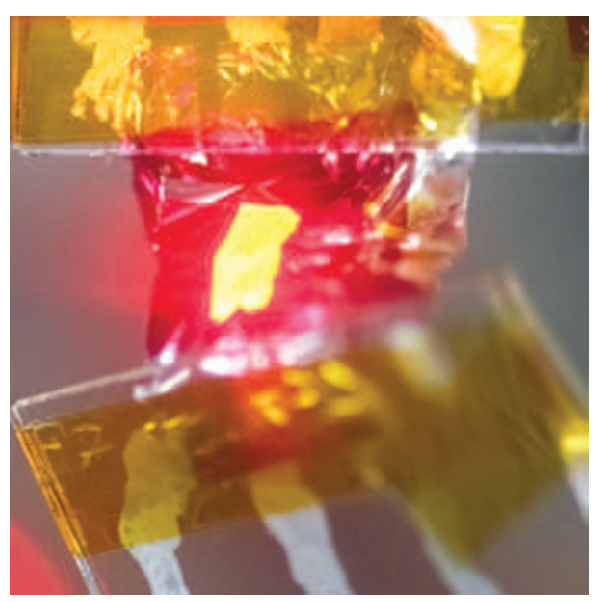

UK talked about the role of spin on organic semiconductor LEDs and solar cells.

According to him, molecular excitons in organic semiconductors show relatively large exchange energies. Consequently, in both LEDs and solar cells, lower-energy triplet excitons are likely to compete with the more desirable spin singlet excitons. It is thus important to understand the behaviour of these excitons, and to design devices and materials so as to maximize the performance.

"In LEDs, in three out of four cases, the formation of an exciton from an electron and a hole is expected to produce a triplet exciton, which is not emissive - only one in four cases will result in the formation of a singlet exciton that does emit. The very high efficiencies now available are achieved by adding a heavy-metal complex, which usually contains iridium as it provides sufficiently strong spin-orbit coupling to permit efficient emission from the decay of the triplet exciton to the ground state by phosphorescence. This was developed by Forrest and Thomson, then both at Princeton," said Friend.

At the conference, Friend described an alternative approach that does not require heavy metals, instead it employs conventional polymer LEDs. The population of triplet excitons increases, but then drops through triplet-triplet collisions, which can generate singlet excitons that can subsequently emit photons, raising the efficiency from $25 \%$ to $40 \%$.

"For photovoltaics, electron-hole capture is a potential problem (as it is in all solar cells). Although the electrons and holes are photogenerated, rather than injected as they are in LEDs, the physics is basically the same - electron-hole collisions should result in recombination, and therefore limit the efficiency of charge extraction. Electronhole capture should still produce triplet states in $75 \%$ of cases, and these triplet states should be very damaging to the photovoltaic performance, because they are low in energy and so are terminal events," said Friend. In his talk, he described recent work by him and his co-workers in which they showed that this does indeed happen for many systems, but, very surprisingly, it does not happen for superior organic photovoltaic devices that contain relatively ordered regions of electron-accepting fullerene. They attributed this to competing kinetics (the energetics remain unfavourable).

"This is very good news for organic photovoltaics because it shows that a big potential problem can be engineered out," said Friend. Their recent work points towards new design rules for suppressing electron-hole recombination in photoconversion systems and for preventing the formation of triplet excitons and thereby enhancing the fluorescence efficiency in organic LEDs.

"I think the recent attention given to spin properties illustrates that when the novel and particular features of the semiconductor physics of organics are understood, new avenues for materials design and device architectures can be developed and can take performance considerably further than at present," Friend told Nature Photonics.

Coincidently, this issue has strong coverage of both polymer solar cells and LEDs. On page 825, Xugang Guo and colleagues report new designs of donor polymers that yield organic solar cells with fill factors approaching 80\% - significantly higher than those of conventional solar cells. On page 811, Matthew White and co-workers report ultrathin, highly flexible and stretchable polymer LEDs that emit red or orange light with a luminance greater than $100 \mathrm{~cd} \mathrm{~m}^{-2}$, which is sufficiently high for indoor applications. On page 817 , Jiajie Liang and colleagues report a stretchable polymer LED that emits light even when subjected to strains as large as $120 \%$, showing potential for realizing foldable and wearable optoelectronics. 